\title{
Crisis y Stakeholders: Un Ejercicio Pedagógico de Percepciones
}

\author{
Orlando E. Contreras-Pacheco ${ }^{\text {a }}$; Leonardo H. Talero-Sarmiento ${ }^{\mathrm{b}}$; Laura Escobar-Rodríguez \\ Universidad Industrial de Santander, Bucaramanga, Colombia \\ ${ }^{a}$ ocontrer@uis.edu.co., ${ }^{b} \underline{\text { leonardo.talero@,correo.uis.edu.co., }{ }^{c} \text { laura.escobar@,correo.uis.edu.co. }}$
}

\begin{abstract}
Resumen
En el presente trabajo se propone evaluar el grado de imparcialidad en la percepción general sobre la importancia de los diversos stakeholders junto con lo que ellos reclaman y afirman. Para ello, se pondrán a prueba los constructos relacionados con la influencia, interés, legitimidad y urgencia en un escenario real de crisis medio-ambiental. El estudio propuesto consiste en la evaluación de percepciones sobre un estudio de caso real, a partir de un análisis de correspondencia múltiple aplicado sobre una muestra representativa de estudiantes profesionales de diferentes nacionalidades en una escuela de negocios Francesa acreditada internacionalmente. A partir de los resultados es posible determinar que, contrario a lo esperado en estudiantes con alta formación en negocios, aún se encuentran ciertos sesgos respecto a la importancia que se le debe dar a cada stakeholder en los constructos analizados.
\end{abstract}

Palabras clave: Stakeholders, gerencia de crisis, legitimidad, estudios de caso.

\section{Introducción}

En el mundo empresarial, los stakeholders de una compañía suelen tener intereses diversos. Sin embargo, ante un contexto de crisis organizacional se espera que dichos intereses sean adecuadamente balanceados teniendo en cuenta los problemas surgidos por la crisis misma (Alpaslan, Green, \& Mitroff, 2009; Bundy, Pfarrer, Short, \& Coombs, 2017). Por ejemplo, los desastres medio-ambientales son una buena demostración de lo que está en juego para los diferentes stakeholders como fruto de las acciones de una compañía (Cho, 2009). El 
impacto medio-ambiental y social derivado de los mismos crea un escenario en donde directivos, empleados, comunidades, medios de comunicación, organizaciones no gubernamentales, gobiernos y sociedades en general sienten, expresan, reclaman y juzgan debido al comportamiento de una compañía transgresora y de sus afectados. Y aunque mucho se ha dicho desde la literatura prescriptiva con relación a la forma ecuánime en la que las organizaciones deben gerenciar dichos stakeholders en momentos de crisis (Alpaslan et al., 2009; Savage, Nix, Whitehead, \& Blair, 1991; Ulmer, 2001), muy poco o nada se sabe sobre lo que los futuros gerentes pueden percibir en dichas situaciones. Una propuesta de medición en un entorno académico parecería propicio para darle respuesta a dicho cuestionamiento.

En el año 2013 ocurrió un derrame de carbón mineral en la costa norte Colombiana en el cual se vio involucrada una importante compañía minera. Ante dicho evento, diversos stakeholders intervinieron con el propósito de determinar la responsabilidad y juzgar el plan de contingencia ejecutado por dicha compañía como producto de la crisis desencadenada. Tomando como referencia dicho caso, el presente trabajo se propone evaluar el grado de imparcialidad en la percepción general sobre la importancia de los diversos stakeholders junto con sus reclamos y afirmaciones, en el marco de los constructos relacionados con la influencia, interés, legitimidad y urgencia. Para ello, a partir de una actividad pedagógica, se realiza un estudio soportado en un análisis de correspondencia múltiple, el cual es aplicado a una valoración realizada por 174 estudiantes profesionales de diferentes nacionalidades y pertenecientes a distintos programas de maestría de una escuela de negocios Francesa acreditada internacionalmente.

A partir de los resultados obtenidos es posible determinar ciertos sesgos respecto a la importancia de cada stakeholder en los constructos analizados. Las implicaciones de dichos hallazgos son discutidas a la luz de los constructos medidos y de la literatura prescriptiva en gerencia de los stakeholders y manejo de crisis.

\section{Marco Teórico}

Según autores como Argandoña (2011) y Green (1993), el concepto general de sostenibilidad se mueve entre dos extremos: un enfoque que reduce la responsabilidad de la organización a la obtención del mayor beneficio posible para sus accionistas (e.g. Friedman, 1970; Jensen, 2002; Sundaram \& Inkpen, 2004) y una segunda que extiende la responsabilidad de la organización de incluir una amplia gama de actores con un interés o participación en esta misma organización, llamados stakeholders (e.g. (Donaldson \& Preston, 1995; Flammer, 2015; Freeman, 1984; Godfrey, 2005). 


\subsection{Stakeholders y Gerencia de los Stakeholders}

En primera instancia, los stakeholders son definidos por Freeman (1984) como "cualquier grupo o individuo que pueda afectar o ser afectado por las acciones de las organizaciones en el cumplimiento de sus objetivos de negocio" (p.46). A diferencia de un enfoque puro sobre los accionistas de una organización, el enfoque hacia los stakeholders implica que las necesidades hacia estos últimos requieran ser balanceadas para una adecuada distribución del valor creado y por ende la obtención de una mayor sostenibilidad empresarial (Phillips, Freeman, \& Wicks, 2005; Smith, 2003).

De esta forma, la gestión de los stakeholders requiere, como punto clave, una atención simultánea a los intereses legítimos de todas las partes interesadas, tanto en el establecimiento de estructuras organizacionales y políticas generales como en la toma de decisiones caso por caso (Donaldson y Preston, 1995). Por ello, la gestión de los stakeholders puede ser concebida como el manejo de las expectativas de las partes involucradas en una relación empresa-stakeholder en aras de responder adecuadamente a los intereses que están en juego para cada una de las partes (Manetti \& Toccafondi, 2012).

Por otra parte, desde muy temprano, autores como Savage et al. (1991) definieron una serie de atributos sobresalientes de los stakeholders para su adecuado manejo. De esta forma puede determinarse el grad de importancia que cada compañia le otorga a cada stakeholder por separado

- La urgencia, que determina como la relación con los stakeholders está marcada por el tiempo y es fundamental para la organización.

- El poder o la ifluencia, que indica si los stakeholders pueden influir en otros para tomar decisiones que no habría tomado por su cuenta.

- El interés, que manifiesta el grado de importancia percibida por parte de los stakeholders con relación a las decisiones y/o acciones de la organización; y

- La legitimidad, en donde los stakeholders tienen una capacidad de influencia moral o legal sobre el comportamiento de la empresa.

\subsection{Crisis y Crisis Organizacionales}

Desde el punto de vista organizacional, las crisis son "eventos de baja probabilidad y alto impacto que amenazan la viabilidad de una organización y se caracterizan por la ambigüedad de causa, efecto y medios de resolución, así como por la creencia de que las decisiones deben tomarse rápidamente" (Pearson \& Clair, 1998, p. 60). Sin embargo, las 
crisis no solo afectan a las empresas, sino también a sus partes interesadas (sus stakeholders), lo que ubica a estos últimos en la posición de jueces reales sobre la existencia de tales eventos. Es por esto que una crisis se puede definir más adecuadamente como "la percepción de un evento impredecible que amenaza las expectativas de los stakeholders y puede afectar seriamente el desempeño de una organización y generar resultados negativos" (Coombs, 2015, p. 3).

\section{EI caso}

A las 11:50 pm del sábado 13 de enero de 2013, como parte de una operación normal en el puerto operado por una importante minera transnacional, y tomando en consideración las malas condiciones climáticas en el área, una de las gabarras de la compañía, cargada con 1,870 toneladas de carbón, fue remolcada y dejada amarrada a una boya de balizamiento proxima a puerto. La idea era esperar a que mejorara el clima antes de reanudar las operaciones. Sin embargo, las tormentas y las olas altas continuaron y el agua comenzó a entrar en la gabarra, lo que provocó que la embarcación se sumergiera. Alrededor de siete horas después, y gracias a la luz del sol, el supervisor matutino pudo observar la situación y activó la alarma de emergencia. Como parte del protocolo de rescate de la compañía, se ordenó el despliegue de las grúas y comenzó a retirar el carbón y arrojarlo al agua hasta el punto en que la barcaza podría volver a cubrir su flotabilidad. Se supuso que toda la carga de la barcaza en el momento del accidente, es decir, 1.870 toneladas, fue arrojada al mar durante ese día como consecuencia de esas acciones y decisiones (Anla, 2013, 2014).

La compañía decidió no revelar el incidente al público inmediatamente después de que ocurriera. Sin embargo, en un giro extraordinario de los acontecimientos, 17 días después, un periodista independiente publicó en su blog personal algunas imágenes del accidente, que fueron rápidamente difundidas por los medios oficiales de comunicación de Colombia. Para colmo, desde ese momento, la companía fue descaradamente engañosa, particularmente al hacer declaraciones claramente contradictorias sobre la cantidad de carbón vertido en el lecho marino (Contreras-Pacheco \& Claasen, 2017). Al final, la compañía fue acusada formalmente, y declarada culpable, de causar una gran catástrofe en el medio ambiente colombiano con sus acciones (Molinski, 2013). 


\section{Metodología}

Para evaluar la perspectiva de los estudiantes respecto a la importancia que se le debe dar a los diferentes stakeholdes ante una crisis, durante el presente trabajo es desarrollado un ejercicio académico que gira en torno al caso descrito con anterioridad. Posteriormente, los estudiantes evaluaron la importancia de los reclamos de cada uno de los stakeholders que intervenían en el caso a la luz de cuatro constructos: Influencia, Interés, Legitimidad y Urgencia. Es necesario resaltar que como son considerados ocho (8) stakeholders, una condición de la valoración dada por los estudiantes es que ésta es jerárquica y, por tanto, un estudiante no puede asignarle en un mismo constructo igual valoración a dos stakeholders diferentes. El formato para la valoración se registra en la Figura 1.

\begin{tabular}{|c|c|c|c|c|}
\hline \multirow[b]{2}{*}{$\begin{array}{l}\frac{\pi}{\mathrm{U}} \\
\frac{\mathrm{T}}{\mathrm{T}} \\
\frac{\mathrm{L}}{0} \\
\text { 을 }\end{array}$} & Legitimidad & Urgencia & Influencia & Interés \\
\hline & $\begin{array}{l}\text { (Teniendo en cuenta lo que } \\
\text { reclaman, ¿qué tan legítimo } \\
\text { considera al stakeholder de } \\
\text { Drummond?) }\end{array}$ & $\begin{array}{l}\text { (Teniendo en cuenta lo que } \\
\text { reclaman, ¿qué tan urgente } \\
\text { considera que los } \\
\text { stakeholders de Drummond } \\
\text { deberian haber actuado?) }\end{array}$ & $\begin{array}{l}\text { (¿Qué tanta influencia cree } \\
\text { que los stakeholders de } \\
\text { Drummond pueden ejercer } \\
\text { sobre la organización?) }\end{array}$ & $\begin{array}{l}\text { (¿Qué tan interesados } \\
\text { considera que los } \\
\text { stakeholders de Drummond } \\
\text { están sobre las actividades } \\
\text { de la Organización?) }\end{array}$ \\
\hline \multicolumn{5}{|l|}{ Clientes } \\
\hline \multicolumn{5}{|l|}{ Gobierno } \\
\hline \multicolumn{5}{|l|}{$\begin{array}{l}\text { Directores } \\
\text { Generales }\end{array}$} \\
\hline \multicolumn{5}{|l|}{ Empleados } \\
\hline \multicolumn{5}{|l|}{$\begin{array}{l}\text { Sociedad en } \\
\text { general }\end{array}$} \\
\hline \multicolumn{5}{|l|}{$\begin{array}{l}\text { Medios de } \\
\text { comunicación }\end{array}$} \\
\hline \multicolumn{5}{|c|}{ ONGs y activistas } \\
\hline Proveedores & & & & \\
\hline
\end{tabular}

Figura 1 Tabla de contingencia del análisis de independencia

Una vez realizado el ejercicio, la valoración obtenida es codificada en tablas de contingencia y analizadas mediante una prueba de correspondencia múltiple, la cual es una técnica descriptiva de reducción de dimensiones de más de dos variables estudiadas. (Peña, 2002). A partir de esta técnica se busca identificar si las variables de interés (Stakeholder, Constructo, Valoración) son independientes y, por tanto, no es posible identificar ningún patrón de respuesta.

La matriz de respuestas (tabla de contingencia múltiple de la frecuencia de respuestas) es comparada con una matriz de respuestas esperada (tabla de contingencia múltiple de la frecuencia de respuesta esperada en caso de presentar variables independientes) mediante una prueba de Chi-cuadrado de independencia. 
Para dicho contraste se calcula el estadístico Chi-Cuadrado mediante la siguiente formula:

$$
\mathrm{X}^{2}=\sum_{i} \sum_{j} \frac{\left(F o_{i j}-F e_{i j}\right)^{2}}{F e_{i j}}
$$

Donde:

$\mathrm{X}^{2}=$ Estadístico Chi - cuadrado

$F o_{i j}=$ frecuencia observada .

$F e_{i j}=$ frecuencia esperada.

Donde el $\mathrm{X}^{2}$ se comporta como una distribución Chi-Cuadrada con $(m-1) *(n-1)$ grados de libertad (Sea $m$ el número de filas y $n$ el número de columnas) (Cuadras, 2014). Para ello, durante el presente trabajo se utiliza el paquete de análisis de datos FactoMineR (Lê, Josse, \& Mazet, 2008), el cual permite generar una tabla de contingencia donde se evidencia la naturaleza independiente de los datos.

\section{Resultados}

Una vez realizado el estudio de independencia mediante la prueba de Chi Cuadrado, se obtiene la Figura 2, en ésta, el tamaño de la celda representa la frecuencia observada de respuestas de cada Valoración, asignada en cada stakeholder para cada variable latente; a su vez, se registra un esquema de colores relacionado con el valor de los residuales de Pearson, donde el color azul indica que el valor observado es mucho mayor que el esperado $\mathrm{y}$, en contraparte, el valor rojo expone que el valor observado es inferior al valor esperado. La presencia de valores por encima y por debajo de los esperados indica la no independencia de las variables y por tanto, la probable presencia de sesgos en las percepciones bajo análisis. Teniendo en cuenta lo anterior y el p-valor inferior a 0.05 (Figura 2), es posible determinar que las variables evaluadas no son independientes y, por tanto, existen patrones de respuesta. 


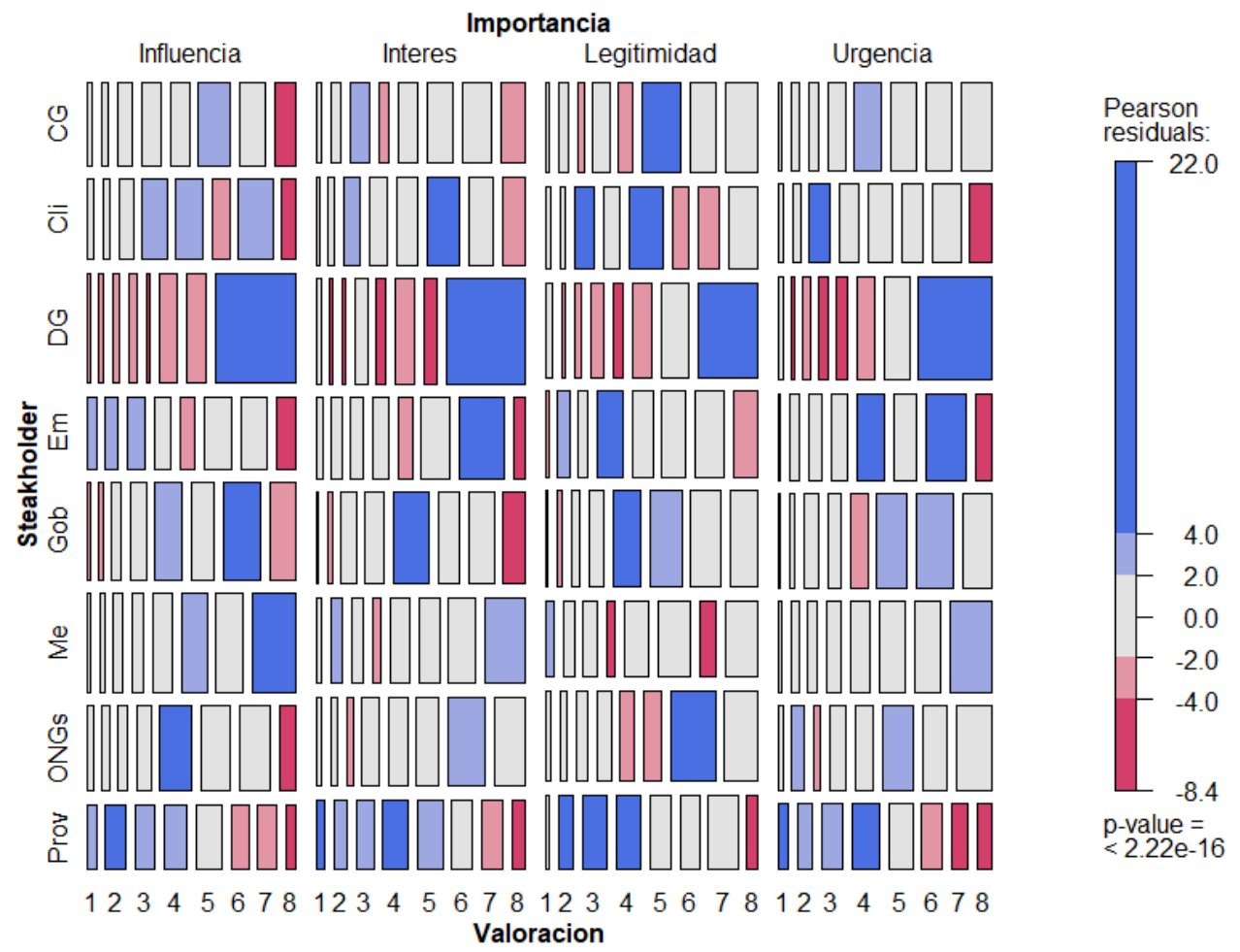

Figura 2 Tabla de contingencia del análisis de independencia

De acuerdo a la percepción de los estudiantes, en el estudio se encuentra que los reclamos realizados por los Directores Generales (DG), tienen un mayor peso en cuanto a la influencia, interés, legitimidad y urgencia, es decir, la opinión de este grupo de interesados es considerada por los estudiantes como muy importante en el mundo empresarial. De otra parte, los estudiantes perciben que la Comunidad en General (CG) presenta un nivel medioalto (rango 6) de importancia en cuanto a la legitimidad e influencia de sus reclamos, se considera además, que la urgencia con la que se deben atender sus reclamos es de nivel medio (rango 5), en cuanto al interés que se le debe dar a este grupo de interesados, pocos estudiantes consideran que son muy importantes (rango 8), asimismo, varios estudiantes consideran que el nivel de interés debe ser bajo (rango 3). Se presentan opiniones divididas en cuanto al nivel de influencia de los reclamos de los Clientes (Cli), si bien gran parte de los estudiantes perciben que este constructo tiene una importancia de nivel medio-alto (rangos 4, 5 y 7), muy pocos estudiantes opinan lo mismo en los niveles medio-alto (rangos 6 y 8); en cuanto a la urgencia con que se deben atender los reclamos de este grupo de 
interesados, pocos estudiantes consideran que deben ser tenidos en cuenta como muy importantes (rango 8), asimismo, varios estudiantes consideran que el nivel de urgencia debe ser bajo (rango 3).

Los reclamos de los Empleados (Em) tienen por su parte un nivel bajo-medio de influencia y legitimidad (rango entre 1 y 4), adicionalmente, no se define una tendencia en cuanto al nivel de interés y urgencia de sus reclamos, lo anterior, debido a que un gran porcentaje de los estudiantes opina que el nivel de estos dos constructos debe ser alto (rango 7); sin embargo, muy pocos estudiantes piensan que el nivel muy alto (rango 8) representa la importancia de los reclamos de este grupo de interesados. Por su parte, los estudiantes consideran al Gobierno (Gob) como un grupo de interesados cuyos reclamos tienen un nivel de urgencia alto (rango 6 y 7) y legitimidad medio (rango 5 y 6), en cuanto al nivel de interés no se define una posición clara debido a que gran parte de los encuestados opina que es de nivel medio (rango 5) y muy pocos les dan un nivel muy alto de importancia (rango 8); ahora bien, en cuanto al nivel de influencia tampoco se determina una tendencia debido a que gran parte de los estudiantes opina que debe darse un nivel de importancia medio-alto (rangos 5 y 7) y muy pocos les dan un nivel muy alto (rango 8).

Los Medios de Comunicación (Me) presentan por su parte un nivel alto en los constructos influencia, interés y urgencia; sin embargo, se considera que el nivel de legitimidad de sus reclamos no es muy alto. De otra parte, las Organizaciones no Gubernamentales (ONGs) presentan un nivel alto en el interés, legitimidad y urgencia y un nivel medio en influencia, de acuerdo a las opiniones de los encuestados. Finalmente, se considera que los reclamos realizados por los Proveedores (Prov) tienen un nivel bajo-medio (rangos 1, 2, 3 y 4) de influencia, interés, legitimidad y urgencia, es decir, no se le da un alto nivel de importancia en el mundo empresarial a este grupo de interesados.

\section{Discusión}

El anterior trabajo abordó la problemática relacionada con la percepción sobre la importancia otorgada por parte de una organización hacia los stakeholders involucrados en una crisis medio-ambiental. Lo hizo a partir de un ejercicio pedagógico de consulta sobre estudiantes de maestría de diferentes nacionalidades a la luz de los constructos influencia, interés, legitimidad y urgencia; tomando como base un caso particular colombiano. Los resultados obtenidos dan cuenta de una clara orientación de los participantes al enfoque hacia los accionistas (Friedman, 1970; Jensen, 2002; Sundaram \& Inkpen, 2004) por encima de un enfoque integral hacia los stakeholders (Donaldson \& Preston, 1995; Flammer, 2015; Freeman, 1984; Godfrey, 2005), pese a que ello represente un retroceso en el camino a la sostenibilidad empresarial (Phillips et al., 2005; Smith, 2003). 
Mas puntualmente, de acuerdo al procesamiento de los datos recolectados, se concluye que la percepción de la importancia sobre la postura de los proveedores y clientes (eslabones de la cadena) no es alta. Lo anterior, en apariencia, arrojaría un otorgamiento de mayor importancia sobre los stakeholders internos, pese a que tomando en consideración el caso tratado, es claro que las expectativas amenzadas, corresponden en mayor medida a los stakeholders externos (medios de comunicación, ONGs y activistas, gobierno, comunidades y sociedad en general) quienes podrían eventualmente afectar los resultados de la compañía causante de la crisis expuesta (Coombs, 2015).

Los resultados obtenidos sugieren también que, no hay imparcialidad sobre cómo perciben que debe ser la importancia de lo que reclaman los stakeholders. Esto se da puesto que en la mayoría de los resultados no se encuentran tendencias definidas, con la salvedad de los Directores Generales ya que estos siempre obtuvieron valores de importancia alta en cuanto la influencia, interés, legitimidad y urgencia. La falta de tendencia o tendencias divididas (según sea el caso), puede indicar que la población bajo estudio presenta ciertas diferencias $\mathrm{y}$ es probable encontrar distintos grupos con posturas diferentes o, dicho de otro modo, distintas corrientes de administración. Por tanto, desde lo metodológico, para futuros trabajos se proponen análisis de reducción dimensional en miras de identificar dichos grupos o perfiles.

Las contribuciones que se derivan del presente trabajo son fundamentalmente dos. En primera instancia, se pone en perspectiva la forma en que, a pesar de la evolución del conocimiento con respecto al modelo sostenible de creación/distribución de valor, parecería que aún existiera una prevalencia de un enfoque puramente utilitarista orientado a los accionistas. Asimismo, derivado de lo anterior, se visualiza un reto claro en términos educacionales. Al parecer los conceptos son adecuadamente entendidos, pero al asumir el rol gerencial, los estudiantes consultados no expresan imparcialidad en la situación tratada. Una retroalimentación adecuada y una concientización real sobre los efectos de dicho enfoque son necesarios en aras de lograr un análisis mas holístico de la situación.

\section{Referencias}

Alpaslan, C. M., Green, S. E., \& Mitroff, I. I. (2009). Corporate Governance in the Context of Crises: Towards a Stakeholder Theory of Crisis Management. Journal of Contingencies and Crisis Management, 17(1), 38-49.

Argandoña, A. (2011). Stakeholder Theory and Value Creation (No. WP-922) (Vol. 3).

Bundy, J., Pfarrer, M. D., Short, C. E., \& Coombs, W. T. (2017). Crises and Crisis Management: Integration, Interpretation, and Research Development. Journal of Management, 43(6), 1661-1692. http://doi.org/10.1177/0149206316680030 
Cho, C. H. (2009). Legitimation Strategies Used in Response to Environmental Disaster: A French Case Study of Total SA's Erika and AZF Incidents. European Accounting Review, 18(1), 33-62.

Contreras-Pacheco, O. E., \& Claasen, C. (2017). Fuzzy reporting as a way for a company to greenwash: perspectives from the Colombian reality. Problems and Perspectives in Management, 15(2), 526-536.

Coombs, W. T. (2015). Ongoing Crisis Communication: Planning, Managing and Responding (Fourth Ed.). Thousand Oaks: SAGE Publications, Inc.

Cuadras, C. M. (2014). Métodos de análisis multivariante. Publicaciones PPU. http://doi.org/10.1017/CBO9781107415324.004

Donaldson, T., \& Preston, L. E. (1995). The Stakeholder Theory of the Corporation: Concepts, Evidence, and Implications. Academy of Management Review, 20(1), 6591.

Flammer, C. (2015). Does Corporate Social Responsibility Lead to Superior Financial Performance? A Regression Discontinuity Approach. Management Science, 61(11), 2549-2568. http://doi.org/10.1017/CBO9781107415324.004

Freeman, R. E. (1984). Strategic Management: A Stakeholder Approach. Management (Vol. 1). http://doi.org/10.2139/ssrn.263511

Friedman, M. (1970). The Social Responsibility of Business is to Increase its Profits. The New York Times, pp. 122-124. New York, NY.

Godfrey, P. C. (2005). The Relationship between Corporate Philanthropy and Shareholder Wealth: A Risk Management Perspective. Academy of Management Review, 30(4), 777-798.

Green, R. M. (1993). Shareholders As Stakeholders: Changing Metaphors Of Corporate Governance. Washington and Lee Law Review, 50(4), 1409-1421.

Jensen, M. C. (2002). Value Maximization, Stakeholder Theory, and the Corporate Objective Function. Business Ethics Quarterly, 12(2), 235-256.

Lê, S., Josse, J., \& Mazet, F. (2008). Package “ FactoMineR ." Journal of Statistical Software, 25(1), 1-18. http://doi.org/10.1007/978-3-540-74686-7

Manetti, G., \& Toccafondi, S. (2012). The Role of Stakeholders in Sustainability Reporting Assurance. Journal of Business Ethics, 107, 363-377. http://doi.org/10.1007/s10551-011-1044-1 
Molinski, D. (2013). Colombia Fines Drummond for Dumping Coal (December 19, 2013). The Wall Street Journal. New York, NY. Retrieved from https://www.wsj.com/articles/SB10001424052702303773704579268432078935884

Pearson, C. M., \& Clair, A. (1998). Reframing Crisis Management. Academy of Management Review, 23(1), 59-76.

Peña, D. (2002). Análisis de datos multivariantes. Book, (December), 515. http://doi.org/8448136101

Phillips, R., Freeman, R. E., \& Wicks, A. C. (2005). What Stakeholder Theory Is Not. Business Ethics Quarterly, 13(4), 479-502. http://doi.org/10.2307/3857968

Savage, G. T., Nix, T. W., Whitehead, C. J., \& Blair, J. D. (1991). Strategies for assessing and managing organizational stakeholders. Academy of Management Executive, 5(2), 61-75. http://doi.org/10.2307/4165008

Smith, H. J. (2003). The Shareholders vs. Stakeholders Debate. MIT Sloan Management Review, 44(4), 85-90.

Sundaram, A. K., \& Inkpen, A. C. (2004). The Corporate Objective Revisited. Organization Science, 15(3), 350-363. http://doi.org/10.1287/orsc.1040.0068

Ulmer, R. R. (2001). Effective Crisis Management Through Established Stakeholder Relationships. Management Communication Quarterly, 14(4), 590-615. 